\title{
Statement on artificial intelligence surgery by women-in-surgery - Italia: can artificial intelligence be the great equalizer in surgery?
}

\author{
Gaya Spolverato ${ }^{1}$, Giulia Capelli ${ }^{1}$, Darya Majidi², Isabella Frigerio ${ }^{3}$ \\ 'Department of Surgical, Oncological and Gastroenterological Sciences, University of Padova, Padova, Padova 35121, Italy. \\ ${ }^{2}$ President of "Associazione Donne 4.0", Livorno, Livorno 57123, Italy. \\ ${ }^{3}$ Department of Hepato-Pancreato-Biliary Surgery, Pederzoli Hospital, del Garda, Verona, Peschiera 37019, Italy. \\ Correspondence to: Dr. Gaya Spolverato, Department of Surgical, Gastroenterological and Oncological Sciences, First Surgical \\ Clinic, University of Padova, Via Giustiniani 2, Padua, Padua 35128, Italy. E-mail: gaya.spolverato@unipd.it
}

How to cite this article: Spolverato G, Capelli G, Majidi D, Frigerio I. Statement on artificial intelligence surgery by women-insurgery - Italia: can artificial intelligence be the great equalizer in surgery? Art Int Surg 2021;1:18-21. https://dx.doi.org/10.20517/ais.2021.06

Received: 22 Jul 2021 Accepted: 19 Aug 2021 Available online: 31 Aug 2021

Academic Editor: Andrew Gumbs Copy Editor: Yue-Yue Zhang Production Editor: Yue-Yue Zhang

\section{INTRODUCTION}

The women working in STEM still represent a minority. Actions undertaken in order to encourage girls to focus on STEM have succeeded in increasing the rate of women obtaining STEM degrees; however, only a minority of them continue to work in the field in which they qualified ${ }^{[1]}$.

In medicine, gender inequalities tend to be more apparent in the surgical specialties; despite an increasing number of women choosing to pursue a surgical career, only a few of them actually achieve leadership positions. In Italy, female surgical residents increased from $33.0 \%$ to $46.2 \%$ in less than a decade; nevertheless women represent only $2.5 \%$ (18/733) of surgical departments chiefs, and $4.9 \%(2 / 41)$ of residency school directors ${ }^{[2]}$. The Association Women in Surgery Italia (WIS Italia ${ }^{\circ}$ ) was founded in 2016 in order to fill the gap of representation within the surgical community. Since the very beginning, the association adopted ambitious goals to promote mentorship and training, and to provide support in the difficult task of creating a woman-friendly work environment. 
Gender bias among physicians, particularly among surgeons, have been repeatedly assessed ${ }^{[3,4]}$. Many health care professionals have been reported to hold implicit and explicit biases associating men with career and women with family ${ }^{[5]}$. Moreover, implicit bias seems to be stronger among residents and women themselves, thus implying that, apart from being relevant and deeply rooted in the society, this issue is unlikely to be resolved in the next few years ${ }^{[6]}$. Some authors have tried to project their attitude and thoughts of male surgeons towards their female counterparts: while $80 \%$ of them consider female surgeons to be as capable as their male colleagues, only $30 \%$ are reported to believe that gender discrimination actually exists in the surgical field ${ }^{[7]}$.

Many possible solutions have been proposed to overcome gender bias in surgery. The possibility to obtain flexible training and career options, the promotion of a less masculine image of surgery itself, the implementation of initiatives to improve work-life balance, and the valorization of same sex mentors and role models have all been proposed as means to make surgery a woman-friendly career ${ }^{[8]}$.

In everyday life, technology could actually represent a powerful mean to overcome inequalities and could help remove some of the barriers that female surgeons face during their professional lives.

Acknowledging this, the aim of this work was to offer a panoramic view on artificial intelligence (AI) applied to the surgical field, and to discuss its potential role as an equalizer for women in surgery.

\section{HOW TECHNOLOGY CAN HELP FEMALE SURGEONS}

AI has been defined as the discipline that studies how to make computers function intelligently and act in the real world ${ }^{[9]}$. As a field of AI, machine learning (ML) aims to reproduce the act of learning and to apply it to machines. A further application of AI is represented by neural networks, a system in which different layers of computer "neurons" perform simple tasks, in order to achieve a final assignment ${ }^{[9]}$. Finally, augmented reality provides an enhanced vision, by superimposing a digital image to the user's view, while virtual reality allows a person to interact with a digitally created environment through the use of dedicated equipment ${ }^{[10]}$.

AI has been employed in various areas in the field of surgery, as a mean to optimize the management of patients and surgical techniques and as a support to training.

AI is currently being tested in clinical practice to aid preoperative diagnosis and staging and to help predict response to treatment, particularly in the field of surgical oncology ${ }^{[1,12]}$. Moreover, some models have been developed with the aim to support clinical decision-making during surgery. For example, Cheng et al. ${ }^{[13]}$ applied ML to laparoscopic cholecystectomy, successfully creating a model for automatic surgical phase recognition, with an overall accuracy of $91.05 \%$. This kind of application could be propaedeutic to the development of software which could actually help surgeons identify anatomical structures and safe planes for dissection ${ }^{[14]}$, and, ultimately, lead to the introduction of collaborative surgical robots which could efficiently assist surgeons during complex procedures ${ }^{[9,15]}$. The widespread adoption of robotic surgery and its latest developments could be of great support for female surgeons, reducing the need for travelling, as already experienced with the diffusion of telemedicine during the COVID-19 pandemic, and contributing to a better work-life balance in a world where women are often both professionals and caregivers. Besides, robotic surgery contributes to remove physical strength barriers, and could allow for female surgeons to continue performing surgery during pregnancy and childbearing. This is particularly relevant, since female surgeons have been reported to postpone pregnancy for various reasons, such as their training and career, which can result in negative impacts on infertility rates and obstetrical complications ${ }^{[16]}$. 
Another important application of $\mathrm{AI}$ is in the field of surgical training. Access to training is pivotal to form and educate the new generation of female surgeons. It is well ascertained that, whatever difference some may argue exist between male and female surgeons concerning surgical skills, it becomes irrelevant following adequate training ${ }^{[17,18]}$. Female surgeons have also been found to be particularly responsive to oneon-one training and instructor feedback, thus furtherly underlying the importance of mentoring ${ }^{[18]}$. Training and surgical mentoring could be provided remotely to surgeons residing in rural areas, which has already been successfully achieved. For example, in Canada a telementoring program was carried on for community surgeons, who were able to perform major surgical procedures with outcomes similar to that of specialized centers ${ }^{[19]}$. This is particularly relevant when considering the possibility to extend training to surgeons residing in low- and middle-income countries, limiting the relevant costs and practical limitations connected to travelling ${ }^{[20]}$. Moreover, augmented reality could provide a versatile and cost-effective tool for training while preserving patients' safety ${ }^{[21]}$. Again, the possibility to train anywhere and anytime could substantially contribute to ameliorating a female surgeon's work-life balance, allowing them to train during pregnancy and to maintain their role as caregivers without sacrificing their careers, and, ultimately, reducing the number of female surgeons who actually quit their job due to the difficulty in balancing their career and family life ${ }^{[22]}$.

\section{CONCLUSION}

In conclusion, AI could be a great instrument to achieve gender equity in surgery, allowing for remote training and facilitating the work of female surgeons worldwide. Since many models and surgical tools are still created and tested mainly on male surgeons, future directions should also consider the development of instruments taking into account gender differences, with the help of many associations which already exist on the national and international territory, such as Women in Health Europe (HIMSS Europe) and Women in Health Italy (HIMSS Italy).

\section{DECLARATIONS}

\section{Authors' contributions}

Made substantial contributions to conception and design of the study: Spolverato G, Capelli G, Majidi D, Frigerio I

\section{Availability of data and materials}

Not applicable.

\section{Financial support and sponsorship}

None.

\section{Conflicts of interest}

All authors declared that there are no conflicts of interest.

\section{Ethical approval and consent to participate}

Not applicable.

\section{Consent for publication}

Not applicable.

\section{Copyright}

(c) The Author(s) 2021. 


\section{REFERENCES}

1. Lancet Digital Health. All things being equal: diversity in STEM. Lancet Digit Health 2020;2:e149. DOI PubMed

2. Available from: http://www.dati.salute.gov.it/dati/dettaglioDataset.jsp?menu=dati\&idPag=62 [Last accessed on 24 Aug 2021].

3. Barnes KL, Dunivan G, Sussman AL, McGuire L, McKee R. Behind the mask: an exploratory assessment of female surgeons' experiences of gender bias. Acad Med 2020;95:1529-38. DOI PubMed

4. Hutchison K. Four types of gender bias affecting women surgeons and their cumulative impact. J Med Ethics 2020;46:236-41. DOI PubMed

5. Salles A, Awad M, Goldin L, et al. Estimating implicit and explicit gender bias among health care professionals and surgeons. JAMA Netw Open 2019;2:e196545. DOI PubMed

6. Kramer M, Heyligers IC, Könings KD. Implicit gender-career bias in postgraduate medical training still exists, mainly in residents and in females. BMC Med Educ 2021;21:253. DOI PubMed PMC

7. Ross SB, Jadick MF, Spence J, DeReus H, Sucandy I, Rosemurgy AS. Men surgeons' perceptions of women surgeons: is there a bias against women in surgery? Surg Endosc 2020;34:5122-31. DOI PubMed

8. Bellini MI, Graham Y, Hayes C, Zakeri R, Parks R, Papalois V. A woman's place is in theatre: women's perceptions and experiences of working in surgery from the Association of Surgeons of Great Britain and Ireland women in surgery working group. BMJ Open 2019;9:e024349. DOI PubMed PMC

9. Ward TM, Mascagni P, Madani A, Padoy N, Perretta S, Hashimoto DA. Surgical data science and artificial intelligence for surgical education. J Surg Oncol 2021;124:221-30. DOI PubMed

10. Badash I, Burtt K, Solorzano CA, Carey JN. Innovations in surgery simulation: a review of past, current and future techniques. Ann Transl Med 2016;4:453. DOI PubMed PMC

11. Kawahara D, Murakami Y, Tani S, Nagata Y. A prediction model for degree of differentiation for resectable locally advanced esophageal squamous cell carcinoma based on CT images using radiomics and machine-learning. Br J Radiol 2021;94:20210525. DOI PubMed

12. Li Q, Xiao Q, Li J, Wang Z, Wang H, Gu Y. Value of machine learning with multiphases CE-MRI radiomics for early prediction of pathological complete response to neoadjuvant therapy in HER2-positive invasive breast cancer. Cancer Manag Res 2021;13:5053-62. DOI PubMed PMC

13. Cheng K, You J, Wu S, et al. Artificial intelligence-based automated laparoscopic cholecystectomy surgical phase recognition and analysis. Surg Endosc 2021. DOI PubMed

14. Madani A, Namazi B, Altieri MS, et al. Artificial intelligence for intraoperative guidance: using semantic segmentation to identify surgical anatomy during laparoscopic cholecystectomy. Ann Surg 2020. DOI PubMed PMC

15. Battaglia E, Boehm J, Zheng Y, Jamieson AR, Gahan J, Majewicz Fey A. Rethinking autonomous surgery: focusing on enhancement over autonomy. Eur Urol Focus 2021;S2405-4569(21)00171. DOI PubMed

16. Todd AR, Cawthorn TR, Temple-Oberle C. Pregnancy and parenthood remain challenging during surgical residency: a systematic review. Acad Med 2020;95:1607-15. DOI PubMed

17. White MT, Welch K. Does gender predict performance of novices undergoing Fundamentals of Laparoscopic Surgery (FLS) training? Am J Surg 2012;203:397-400; discussion 400. DOI PubMed

18. Ali A, Subhi Y, Ringsted C, Konge L. Gender differences in the acquisition of surgical skills: a systematic review. Surg Endosc 2015;29:3065-73. DOI PubMed

19. Anvari M, Manoharan B, Barlow K. From telementorship to automation. J Surg Oncol 2021;124:246-9. DOI PubMed

20. Pears M, Konstantinidis S. The future of immersive technology in global surgery education. Indian J Surg 2021;1-5. DOI PubMed PMC

21. Ip M. Technology not policy will help drive female consultant numbers higher. Bulletin 2020;102:134-7. DOI

22. Maehara T, Kamiya K, Fujimaki T, et al; Gender Equality Committee of the Japan Neurosurgical Society. A questionnaire to assess the challenges faced by women who quit working as full-time neurosurgeons. World Neurosurg 2020;133:331-42. DOI PubMed 